\title{
Le marché de l'office et des capitaux dans la France de l'Ouest
}

À propos de la thèse de François Caillou, Une administration royale d'Ancien Régime : le bureau des finances de Tours, Tours, Presses universitaires François-Rabelais, 2 vol., 2005, 496 et 436 p.

\section{Dominique Le Page}

\section{(e) OpenEdition \\ 1 Journals}

Édition électronique

URL : http://journals.openedition.org/abpo/606

DOI : $10.4000 /$ abpo.606

ISBN : 978-2-7535-1505-5

ISSN : $2108-6443$

\section{Éditeur}

Presses universitaires de Rennes

\section{Édition imprimée}

Date de publication : 30 mars 2007

Pagination : 199-210

ISBN : 978-2-7535-0452-3

ISSN : 0399-0826

\section{Référence électronique}

Dominique Le Page, "Le marché de l'office et des capitaux dans la France de l'Ouest », Annales de Bretagne et des Pays de l'Ouest [En ligne], 114-1 | 2007, mis en ligne le 30 mars 2009, consulté le 23 septembre 2020. URL : http://journals.openedition.org/abpo/606 ; DOI : https://doi.org/10.4000/abpo. 606 


\title{
Note critique \\ Le marché de l'office et des capitaux dans la France de l'Ouest
}

(À propos de la thèse de François Caillou, Une administration royale d'Ancien Régime : le bureau des finances de Tours, Tours,

Presses universitaires François-Rabelais, 2 vol., 2005, 496 et 436 p.)

\author{
Dominique Le PAgE \\ Université de Nantes
}

Cet ouvrage, qui est la version abrégée d'une thèse réalisée sous la direction de Claude Petitfrère à l'université François Rabelais, porte sur le bureau des finances de Tours, de 1577, date de la création de cette institution par Henri III, à la Révolution. Faute de sources suffisantes et malgré un louable effort pour exploiter les documents disponibles qu'il s'agisse des arrêts du conseil du règne de Henri IV publiés par Noël Valois ou des archives départementales (18 dépôts consultés) et nationales, l'essentiel du propos concerne la période qui va du règne de Louis XIV à 1789. Après le travail pionnier de Jean-Paul Charmeil (Les trésoriers de France à l'époque de la Fronde. Contribution à l'histoire de l'administration financière sous l'Ancien Régime, 1964), et les études de Philippe Rosset sur le bureau de finances de Lille, d'Emmanuelle Ashta sur celui de Paris, de Valérie Brousselle-Basques sur celui de Montauban ou de Françoise Bayard sur celui de Lyon, cette recherche vient compléter la connaissance que l'on peut avoir de cette institution et de son personnel, en manifestant le souci particulier d'étudier au plus près son activité et de discuter l'idée de son déclin au cours de l'époque moderne.

L'ouvrage se compose de deux parties divisées chacune en quatre chapitres. La première est consacrée à l'étude du fonctionnement du bureau des finances et la seconde au personnel. Après un rappel de l'histoire de l'administration financière depuis le XIV ${ }^{\mathrm{e}}$ siècle et des réformes financières de François ${ }^{\text {er }}$ et de Henri II qui ont entrainé une réduction drastique des pouvoirs des généraux de finances puis une provincialisation de leur domaine d'intervention par la mise en place (1542 et 1551) de 17 généralités, le premier chapitre présente le cadre et le fonctionnement du bureau des finances de Tours à partir de juillet 1577. À cette date, Henri III crée 
dans cette ville, comme dans les 16 autres chefs-lieux de généralité, des offices de trésoriers généraux de France dont les titulaires doivent, de façon collégiale, gérer le domaine royal et les impôts. À Tours, la composition du bureau est fixée à 5 trésoriers généraux de France, un greffier, quatre huissiers audienciers. Sa compétence s'étend à trois provinces - l'Anjou (39,8 \% du ressort territorial avec 637 paroisses), le Maine (32,5\% et 562 paroisses) et la Touraine (27,7 \% avec 422 paroisses), ce qui, avec un ressort d'une superficie de $26504,5 \mathrm{~km}^{2}$, le place au $5^{\mathrm{e}}$ rang français derrière Montpellier, Rennes, Bordeaux et Auch.

Comme ailleurs, les débuts du bureau des finances sont difficiles. Supprimé en 1583, sous la pression des Ligueurs qui veulent réduire l'État d'offices, il est rétabli deux ans plus tard mais il doit évoluer, jusqu'à la fin du règne de Henri IV, dans un climat d'incertitude et ses membres doivent verser à plusieurs reprises des sommes substantielles pour être maintenus dans leurs fonctions. Le bureau souffre en outre de sa position excentrée dans la généralité et de la concurrence de villes plus peuplées comme Angers, qui conteste à Tours sa suprématie. L'adhésion du Bas-Maine et du nord de l'Anjou à la Ligue (1589-1595) peut s'interpréter ainsi comme une tentative d'émancipation des bourgeoisies robines de ces secteurs (Laval, Château-Gontier, Craon et à un moindre degré Le Mans) par rapport à la ville royale. Plus gravement, un lobby angevin, composé de membres de la municipalité, du présidial et de l'élection tente à trois reprises - 1624, 1638, 1645 - d'obtenir un bureau des finances. Les efforts conjugués des trésoriers de Tours et du corps municipal où siègent plusieurs d'entre eux (dont René Sain) font échouer ces projets. Il n'y a plus de menace sérieuse dans ce domaine jusqu'à la fin de l'Ancien Régime, le projet au printemps 1771 de Louis-Stanislas Xavier, petit-fils de Louis XV, de créer une Chambre des comptes pour son apanage de l'Anjou et du Maine tournant vite court.

Conforté dans son implantation tourangelle, le bureau des finances voit son personnel s'étoffer progressivement, avec des pointes, comme dans le reste du royaume, sous les règnes de Henri III, de Louis XIII à partir des années 1620 (13 offices créés) et de Louis XIV, dans les années 1690-1700. Jusque dans la première moitié du XVII ${ }^{\mathrm{e}}$ siècle, le pouvoir royal actionne plusieurs leviers pour créer de nouveaux offices. Il joue tout d'abord de la menace de suppression de l'institution ou de démembrement de son ressort : ainsi en 1585, le rétablissement du bureau de Tours est accompagné de l'introduction de deux charges de trésoriers. De même en 1626, la renonciation du pouvoir à créer un bureau des finances à Angers a pour conséquence la mise en vente de deux autres offices. L'autre procédé utilisé est l'accroissements de juridiction : en 1581, l'attribution au bureau du jugement souverain des appels des sentences des élus pour affaires n'excédant pas 10 l. et la vérification des comptes des deniers communs et d'octroi (qui était du ressort des Chambres des comptes) s'accompagne de la création d'une $6^{\mathrm{e}}$ charge de trésorier de France et d'un office de président. Il en va de même en 1621 quand le bureau se voit confier la tâche de vérifier les 
comptes des ponts et chaussées (deux nouveaux offices de trésoriers), et plus encore, en 1627, quand la monarchie lui accorde le jugement des causes domaniales et de voirie et qu'il est pourvu d'un véritable ministère public, ce qui permet l'introduction de dix offices de procureurs dont seulement cinq trouvent preneurs. L'entrée de la France dans la guerre de Trente ans en 1635 se traduit par la création de 4 présidents et intendants généraux des finances qui doivent se substituer aux commissaires départis qui ont commencé à faire leur apparition dans les généralités. Sous Louis XIV, le gouvernement ne s'embarrasse plus de telles précautions et les créations dévoilent leur caractère purement financier comme celle d'un chevalier d'honneur en 1702. Au total de 45 officiers en 1661, le personnel passe à 52 en 1715 avant de retomber à 44 en 1789. En 1714, 2,6\% de la taille et des impôts accessoires collectés dans la généralité servent à rétribuer les officiers, $1,8 \%$ en 1769 .

Si les trésoriers de France sont les éléments moteurs de l'institution, F. Cailllou montre bien - et c'est l'un des mérites de son étude - le rôle décisif joué par le personnel subalterne qu'il s'agisse de ceux qui en font partie comme les greffiers parmi lesquels figure un temps le célèbre Charles Paulet, des huissiers qui font les commandements et les exploits ordonnés par compagnie ou des officiers ministériels comme les procureurs dont la tâche consiste à représenter les individus et les personnes morales qui requièrent l'intervention du bureau des finances et les plaideurs qui y portent une cause ou doivent s'y défendre. Malgré l'augmentation du nombre des gens qui y travaillent, le bureau tarde à s'établir dans un local bien à lui. Après une installation au couvent des Augustins puis au vieux palais de Tours où il côtoie le bailliage-présidial, il aménage en 1665 dans un ancien hôtel aristocratique racheté aux Rohan-Guéméné. Ce n'est qu'en 1753, après un accord avec les chanoines de Saint-Martin, qu'il bénéficie d'un logis neuf près de la porte de l'horloge, dans un quartier huppé où vivent les magistrats du présidial, de riches ecclésiastiques, des négociants et habitants de Saint-Domingue. Ces déménagements successifs résument l'histoire de l'institution de façon quelque peu paradoxale : sans local spécifique quand ses pouvoirs étaient les plus étendus, elle est dotée d'un bâtiment fonctionnel au temps de la monarchie administrative, alors qu'elle n'est plus qu'un rouage secondaire de l'appareil d'État.

Les chapitres II et III de la deuxième partie décrivent avec précision ce recul d'influence du bureau dans les secteurs des finances et de l'administration domaniale. Cette étude est précédée d'une réflexion sur la notion de service sous l'Ancien Régime. Le bureau des finances de Tours assurait 130 séances de travail par an à raison de trois audiences par semaine (lundi, mercredi et vendredi) à l'exception d'une période de vacances du 8 septembre au 11 novembre où il ne siégeait que le vendredi. Les mois les plus actifs étaient janvier et juillet (avec plus de 13 séances en moyenne) alors que les plus " calmes " étaient logiquement ceux de septembre et d'octobre (4). Combattant l'idée d'un absentéisme systématique des officiers sous 
l'Ancien Régime, F. Caillou montre qu'ils ne pouvaient considérer leur charge comme une sinécure. Ils ne pouvaient en premier lieu ignorer totalement les règlements royaux, - notamment celui de 1627, renouvelé en 1671 -, qui leur faisaient obligation de résider au moins trois mois au cheflieu de leur généralité et auxquels seuls ceux qui étaient au service du roi pouvaient espérer se soustraire. Ils devaient ensuite, pour des raisons pécuniaires, être présents à 36 séances au moins s'ils voulaient prendre part aux épices. Ces contraintes expliquent que la majorité des officiers étaient présents lors de 50 à 60 réunions et que chaque année seuls 7 à 10 officiers ne faisaient pas leur service. Le bureau pouvait donc fonctionner correctement grâce notamment aux officiers originaires de Tours et aux allogènes qui s'y installaient à demeure, au moins durant le temps de leur carrière. Les moins assidus n'étaient pas inutiles et pouvaient représenter le bureau sur leur lieu de résidence ou servir les intérêts de leurs collègues auprès du gouvernement, s'ils étaient employés dans les bureaux des ministères.

Les uns et les autres furent impuissants face à la remise en cause de leurs attributions à partir du règne de Louis XIII. C'est sur le plan financier que celle-ci fut la plus nette. On sait que dans ce domaine, les trésoriers avaient pour tâches de répartir la taille entre les élections après avoir fait une chevauchée à la fin de l'été dans la généralité, d'établir un état des finances de celle-ci et de contrôler les comptes des receveurs. Aucun fonds ne pouvait être employé sans une ordonnance de leur part et ils pouvaient disposer des sommes non prévues par le budget (excédents, rentrées imprévues...) pour répondre aux besoins les plus urgents (paiement de créanciers de la monarchie, de gages aux officiers locaux ou d'intérêts en retard des rentiers de l'État...) et répartir les taxations extraordinaires. Dans le domaine de la fiscalité indirecte, ils avaient pour mission de procéder aux adjudications des baux des aides et des traites dans l'auditoire des élections et d'enquêter sur les décharges demandées par les fermiers. À partir de 1578, ils reçurent d'importantes prérogatives en matière de finances urbaines en se voyant charger de bailler des fermes des deniers d'octroi, de contrôler l'emploi fait par les municipalités des sommes prélevées et de visiter les ouvrages édifiés. Dans le domaine militaire enfin, ils désignaient les lieux d'étapes en accord avec le gouverneur, s'assuraient de la collecte des sommes nécessaires pour le ravitaillement des troupes par les autorités locales et veillaient au sort des prisonniers de guerre.

La plupart de ces prérogatives sont battues en brèche à partir des années 1620. Dès 1629, le conseil royal s'attribue l'adjudication des baux des impôts indirects, ne laissant plus aux trésoriers que la tâche de les enregistrer et de recueillir les plaintes des habitants. Placés sous l'autorité du surintendant des finances, ils voient leur marge de manœuvre réduite par les intendants. En 1634, le maître des requêtes Jean d'Estampes de Valençay et Jean de Bragelongue viennent à Tours pour opérer un nouveau régalement des tailles dans la généralité. En 1637, Jean Martin de Laubardemont, qui a une commission d'intendant de justice, police et finances, procède à la levée d'un emprunt sur Tours (250000 écus) à la 
place des trésoriers et en 1641, Jean-Jacques Renouard se substitue également à eux pour organiser la levée d'une subvention générale du vingtième sur les denrées et marchandises entrant dans les villes de la généralité. L'estocade est portée en 1642-1643 (déclaration du 16 avril 1643) quand les intendants se voient attribuer l'essentiel de la répartition et de la surveillance de la levée des tailles, taillons, crues et subsistance des troupes. Les commissions de taille sont désormais envoyées au bureau des finances et à l'intendant. La fonction de trésorier de France est alors vidée de l'essentiel de son contenu. Dans cette évolution, la Fronde n'offre qu'un répit provisoire : si les intendants sont supprimés (déclaration du 11 juillet 1648), les trésoriers ne parviennent pas vraiment à rétablir leur autorité et ils voient leur action entravée par les élus qui acceptent mal leurs interventions pour présider les opérations de répartition de la taille. Avec le retour au calme, les intendants font leur retour dans les généralités sous le nom de commissaires départis et en Touraine, c'est significativement Denis de Heere, qui est rétabli dans une fonction qu'il avait déjà exercée de 1641 à 1647.

Sur tous ces points, F. Caillou restitue pour la généralité de Tours une chronologie que l'on connaît bien par ailleurs. Il se montre plus original quand il essaie de réfléchir aux raisons qui ont amené la monarchie à déposséder les trésoriers de France de leurs pouvoirs. Rejetant l'hypothèse selon laquelle on ait voulu les punir pour leur insubordination - en 1640, ils ont contribué à lutter contre une émeute populaire à Tours et s'ils ont été moins actifs en 1643, c'est que l'intendant était alors présent dans la généralité-, ou pour leur malhonnêteté, il analyse la critique faite aux trésoriers de sous-imposer les élections dont ils étaient originaires. Grâce à une étude de la pression fiscale pesant sur les différentes parties de la généralité, il montre que les inégalités sont dues en fait au poids des héritages - les guerres de Religion ayant amené à ménager le Maine, le nord de l'Anjou pour obtenir leur ralliement à partir de 1594 - et à une mauvaise connaissance des réalités démographiques et économiques plus qu'à un quelconque favoritisme. Les trésoriers de France ont été victimes surtout de leur trop grande lenteur à faire rentrer l'impôt. Contraints de respecter les règles coutumières, ils n'ont pu faire face aux exigences de la monarchie qui ont connu, dans la généralité de Tours, une progression encore plus spectaculaire qu'ailleurs puisque la taille et ses accessoires sont passés de 566493 l. en 1636 à 1464231 l. en 1637 pour atteindre un record de 5233749 l en 1643 faisant d'elle l'une des plus taxées du royaume. Les intendants, en faisant la preuve de leur efficacité, avec l'appui il est vrai de la force armée, les ont réduits à un rôle secondaire.

À partir de 1661, les trésoriers n'ont gardé quelque pouvoir sur le plan financier qu'en devenant leurs auxiliaires. S'ils ont continué à effectuer leurs chevauchées, seul l'un d'entre eux, désigné d'abord par le bureau des finances puis choisi par l'intendant, a pu assister ce dernier lors du département des tailles entre les paroisses. Dans les chefs-lieux d'élection, ils ont été concurrencés par les subdélégués qui ont mis en place un réseau 
parallèle d'information du pouvoir. Ce déclin de leur rôle sur le plan fiscal a eu pour corollaire de modifier la nature des causes qu'ils ont été amenés à juger dans le domaine du contentieux, les litiges en matière d'impôt cédant le pas aux problèmes de paiement de gages ou de rentes. Si les questions des deniers d'octroi ont continué à leur être soumises, cela ne saurait masquer le fait que sur ce point aussi, ils ont vu leurs prérogatives rognées du fait que la monarchie a fait main basse sur la moitié des sommes perçues, d'abord de façon ponctuelle en 1662, puis à perpétuité en 1681, et que les intendants ont reçu la tâche de procéder à l'adjudication des baux de l'autre moitié au chef-lieu de chaque généralité (1689). Après être parvenus, dans un premier temps, à éviter un transfert des opérations d'affermage à l'hôtel de ville de Tours en acceptant notamment la création d'un office de premier président (1691), ils ont dû céder quand ce dernier n'a plus été pourvu de titulaire (1719); le fait que leurs pouvoirs en la matière aient été préservés dans les chefs-lieux d'élection n'a pu compenser leur mise à l'écart dans le chef-lieu de la généralité. Dans ce contexte difficile, la monarchie ne les a pas complètement mis de côté et leur a confié quelques missions ponctuelles comme celle, de 1663 à 1665, de vérifier les titres des officiers de judicature. Cette enquête, qui sert de base à l'établissement à la nouvelle tarification des offices par Colbert (1665), constitue aujourd'hui une source exceptionnelle pour les historiens en leur en donnant à connaître le nombre de charges existant dans le royaume, généralité par généralité, leur prix officiel et leur cours sur le marché.

Mis à l'écart sur le plan fiscal, les trésoriers ont vu également leurs prérogatives en matière domaniale réduites, même s'ils n'y avaient jamais joué un rôle considérable. À partir de 1629, le Conseil s'est attribué la mise aux enchères des droits domaniaux qu'ils effectuaient depuis 1577; sous Colbert, ils ont été évincés de la surveillance des châteaux et au XVIII siècle, les devis des réparations des autres bâtiments royaux leur ont été enlevés au profit des ingénieurs des ponts-et-chaussées, les intendants intervenant ensuite pour procéder aux adjudications. S'ils ont conservé le pouvoir de lutter contre les usurpations et de recevoir les hommages des vassaux du roi, leur efficacité sur ce point a été limitée du fait qu'ils n'ont jamais disposé des informations pour opérer les contrôles indispensables, que nombre de bénéficiaires d'aliénations du domaine ne leur présentaient pas leurs lettres de don et que la réformation des terriers sous Colbert fut confiée aux lieutenants généraux des bailliages et présidiaux (1664) puis aux intendants (1673).

Ce constat, globalement négatif, contraste avec la hausse de l'activité du bureau, perceptible notamment grâce à l'examen du nombre de sentences rendues en audience qui connaît une augmentation de $200 \%$ du ministère de Colbert à la fin du règne de Louis XV. À travers cette évolution se lit la mutation qui s'opère au $\mathrm{XVIII}^{\mathrm{e}}$ siècle dans la fonction des trésoriers de France qui, d'acteurs de l'appareil financier, deviennent des spécialistes de la voirie et de l'urbanisme, point qui fait l'objet du quatrième 
chapitre de la première partie. Écartés par l'arrêt du 21 octobre 1669 de la direction des ponts et chaussées et de la gestion des voies royales qui revient là encore à l'intendant, ils assurent leur survie en prenant en charge l'administration du réseau secondaire et des voies urbaines. Capitale à cet égard est l'ordonnance prise par le bureau de finances de Tours le 28 janvier 1737 qui entraine la mise en place en Touraine et en Anjou d'un réseau d'agents - les commis voyers - chargés de faire respecter les règles en matière de grande et de petite voirie. Grâce à une vigilance accrue, les trésoriers contribuent à l'entretien des principales routes, sans avoir le pouvoir toutefois d'en faire construire de nouvelles, et à améliorer la circulation dans les agglomérations (pavage des rues, alignement des maisons...). En mobilisant toutes les sources disponibles, François Caillou parvient à montrer que les trésoriers de France ont su se rendre utiles au siècle des Lumières et à s'ouvrir aux idées nouvelles, faisant la preuve ainsi d'une belle vitalité qui contredit l'image un peu terne que l'historiographie donnait traditionnellement d'eux. Leur connaissance insuffisante des populations, leur manque de formation, les querelles de juridiction avec le parlement de Paris, le refus des grands seigneurs et des autorités municipales de leur obéir, ont toutefois fini par réduire leur capacité d'intervention à la veille de la Révolution.

Le deuxième volume s'intéresse au personnel des bureaux de finances et s'interroge tout d'abord (chapitre V) sur le fait de savoir s'ils appartenaient à une élite sociale en prenant comme paramètres, dans une logique qui paraît parfois discutable, leur origine géographique et sociale, leurs alliances et leur descendance ainsi que le regard que la société portait sur eux. On y apprend que le personnel subalterne (procureurs, huissiers) se recrutait, à l'instar de ce que l'on constate dans la plupart des institutions d'Ancien Régime, à une très nette majorité dans un rayon limité (86,8 \% de Touraine) alors que les officiers supérieurs, pour lesquels on a surtout des informations pour la période après 1650, avaient une origine plus variée, avec une prépondérance d'individus issus de la généralité (Touraine : $67,3 \%, 20 \%$ d'Angevins, $19,1 \%$ du Maine) et une forte minorité d'extérieurs (13,6 \%), où l'on comptait surtout des Parisiens. Sur le plan social, on constate un recul de la part des nobles qui passe de $43 \%$ avant 1700 , à $13,8 \%$ après. Le Bureau n'a été un club réservé au second ordre que pendant la période 1577-1583 et il a accueilli ensuite surtout des nobles récents. Pour espérer y entrer, il fallait toutefois appartenir à une famille d'un statut social élevé, qui s'était détournée des métiers mécaniques depuis au moins deux générations. La majorité des trésoriers étaient les descendants d'officiers royaux ou seigneuriaux (particulièrement pour ceux qui venaient du Maine) avec une tendance là aussi à une certaine dégradation puisque l'on ne rencontre plus, après 1700 , de fils de membres de cours souveraines ni de maison du roi ou des princes. On assiste en revanche à une augmentation de la part des descendants de marchands et d'entrepreneurs. Entrés en deux vagues - la première dans les années 1620-1640, la seconde après la guerre de Guerre de Hollande -, mais sans faire l'acquisition massive d'of- 
fices nouveaux, ce qui peut paraître paradoxal, ils ont fait jeu égal avec les robins dans les trente dernières années de l'Ancien Régime, confirmant l'idée que les compagnies de finances ont été plus ouvertes sur le plan social que les institutions judiciaires. L'importance du secteur manufacturier de la soie, le fait aussi que Tours ait été longtemps l'un des centres financiers du royaume ont de toute façon empêché qu'il y ait une fracture trop nette entre les gens de justice et les marchands qui entretenaient des relations sur les plans familial et financier. L'observation des alliances des trésoriers et de leur descendance confirme leur appartenance à l'élite. Ils épousaient majoritairement des filles d'officiers (61,9\% des beaux-pères) puis de marchands $(17,6 \%)$ et de bourgeois et de sieurs $(9,1 \%)$. Leurs enfants se distribuaient, sans surprise, entre le monde de l'office, le clergé et l'armée (surtout à partir du règne de Louis XIV). Seule une minorité succédaient à leur père au bureau des finances, ce qui contredit l'idée que les individus y entraient surtout pour s'anoblir sur deux générations (18,5\% des lignages bénéficièrent de cette procédure).

Bien que privilégiés (la moitié des trésoriers ont des fortunes qui dépassent les 100000 l.), comme le confirment les sommes qu'ils paient pour le droit de marc d'or ou les tarifs auxquels ils sont soumis pour la capitation (ils sont dans la dixième classe taxés à 120 l.), les trésoriers de France ont eu du mal à s'imposer sur la scène locale, particulièrement lors des grandes manifestations publiques. Contraints de laisser la préséance au présidial et au corps municipal du fait de leur création tardive, ils préfèrent ne pas comparaître lors des grandes processions religieuses ni lors des entrées royales Cette discrétion ne les empêche pas d'être en butte aux attaques des officiers du présidial qui veulent avoir une position privilégiée dans les commissions réunies pour la gestion de l'hôpital général de la Charité et qui veulent les contraindre, en 1682, d'assister à la procession du SaintSacrement afin de les mettre en position d'infériorité. Le pouvoir royal, pris comme arbitre, tranche en faveur des trésoriers, mais sans pouvoir faire appliquer sa volonté. De ce fait, les trésoriers, dont la préséance est même contestée par les fabriciens des paroisses, cessent, à partir de 1720, de comparaître en corps dans les églises à l'occasion des funérailles de leurs collègues, pour lesquels ils doivent faire célébrer des services privés. Sur le plan cérémoniel, le bureau des finances est donc une institution invisible et contestée à Tours, ce qui ne l'empêche pas d'envoyer des délégations lors du passage dans la ville de grands personnages de la famille royale ou de l'État.

Le chapitre VI intitulé " l'office et la carrière " s'intéresse à la carrière du personnel du bureau des finances. La carrière des trésoriers était d'une durée moyenne de 21 ans 6 mois (28 ans avant 1700, 18 ans en moyenne après). La plupart des charges étaient mises en vente après la mort des titulaires et revenaient à des membres de la famille ou à des proches quand elles n'étaient pas, nouveautés du XVIII ${ }^{\mathrm{e}}$ siècle, vendues par voie d'affiche ou par annonce dans les journaux. Après une hausse dans la première moitié du XVII ${ }^{\mathrm{e}}$ siècle (vente entre 60000 et 70000 l.), les prix des offices de tré- 
sorier baissent sous le ministériat de Colbert du fait de la tarification établie par ce dernier en 1665 (40000-30000 l), avant de connaître une reprise de la fin du règne de Louis XIV jusqu'aux années 1760 (50000 l.) puis un tassement à la veille de la Révolution. La valeur des autres charges connaît la même évolution d'ensemble avec dans le cas de l'office d'huissier collecteur une grande sensibilité à l'activité du Bureau puisque les prix passent de 2500 l. - 2800 l. en 1726 à 7000 l. en 1769, lors de l'extension des attributions en matière de voirie (rechute à 3000 l. en 1779).

Après la délivrance des lettres de provision par les chancelleries, les trésoriers devaient être reçus par la Chambre des comptes de Paris qui les soumettait à une information de vie et mœurs, vérifiait qu'ils avaient bien les 25 ans requis et les interrogeait, de façon formelle, sur les matières domaniales et fiscales. Les trésoriers ne devaient pas être gradués (un quart l'était contre $40 \%$ à Lille) et la formation de la plupart d'entre eux se limitait à celle qu'ils avaient reçue en collège. Ils devaient être installés ensuite au bureau des finances par leurs collègues. Les délais de réception étaient courts - moins de six mois en général - et les refus étaient rares : ils concernaient ceux dont les parents étaient demeurés redevables à l'égard du roi ou, fait exceptionnel, ceux qui étaient trop marqués politiquement (comme Denis Charpentier, secrétaire de Richelieu, qui vit sa candidature repoussée en 1626 en raison probablement du ralliement d'une majorité des trésoriers à Gaston d'Orléans).

La plupart des officiers entraient dans les bureaux des finances pour bénéficier des privilèges très recherchés qui étaient attribués à leurs membres comme l'anoblissement graduel (qui est étendu au greffier en chef en 1695), l'emploi d'épithètes d'honneur (monsieur maître au XvII siècle, messire au $\mathrm{XVIII}^{\mathrm{e}}$ siècle), le droit de commitimus, l'exemption de la taille et des accessoires, des étapes (mais pas du logement des gens de guerre), des lods et ventes. Pour la gabelle, ils bénéficiaient, depuis un édit d'avril 1519, du droit de prendre au grenier dont ils dépendaient le sel pour leur approvisionnement au prix du marchand. Signe de la fragilisation de leur position et de l'accroissement de la pression fiscale, certains de leurs privilèges ont été remis en cause par les fermiers et les partisans ou réduits à partir du règne de Louis XIV, particulièrement en ce qui concerne les aides, les octrois, la gabelle pour laquelle leur exemption est finalement établie à 2 minots. La réforme L'Averdy de 1765 menace un temps leur droit de ne pas être institué maire ou échevin sans leur consentement en décidant que dans toute ville où siège un bureau des finances, un des membres devait être élu au rang de notable. Si le pouvoir renonce finalement à cette réforme, les luttes constantes qu'ils doivent mener pour la défense de leurs privilèges révèlent leur position ambiguë dans l'appareil d'État où ils ne parviennent jamais, malgré leurs prétentions, à être mis sur le même pied que les cours souveraines. Cette faiblesse tient peut-être en partie aux carrières menées par les officiers, qui sont décrites ensuite par F. Caillou. La moitié d'entre eux n'ont pas débuté leur vie professionnelle au bureau, ce qui explique un âge moyen assez élevé (34 ans) à leur accession à l'office : ils ont été préalablement offi- 
ciers royaux ou seigneuriaux, comptables, marchands et entrepreneurs, militaires ou, dans quelques cas, ecclésiastiques. Si la plupart ont cessé leur activité pour devenir trésoriers, cela n'a pas été le cas de tous au XVIII ${ }^{\mathrm{e}}$ siècle. Quelques uns ont cumulé leur charge avec un office de conseiller au présidial ou poursuivi une activité de financier alors que certains marchands ont continué à pratiquer le négoce, portant ainsi atteinte à la cohésion du corps. Ce défaut a été encore plus net au sein du personnel subalterne, particulièrement chez les procureurs, qui n'ont pas hésité à se mettre au service de plusieurs cours. Du fait de leur entrée dans l'institution à un âge avancé, la plupart des officiers sont morts en charge. Ceux qui ont démissionné n'ont pas exercé d'autres activités après et le bureau des finances n'a pas été une pépinière de serviteurs royaux.

En s'inspirant des idées de David Bien, François Caillou montre dans le chapitre VII comment le bureau est devenu, à l'instar des corps constitués, un organe d'emprunt étatique par lequel la monarchie, en imposant aux officiers l'achat de suppléments de gages, d'épices a fait d'eux des relais dans la chaîne du crédit. Grâce à un recensement des prélèvements qu'ils ont subis, il montre qu'ils ont fourni à la monarchie plus de 3 millions de livres entre 1610 et 1788 . Les taxes les plus nombreuses ont été levées bien entendu sous le règne de Louis XIV et c'est en 1673 que le bureau s'est engagé pour la première fois de façon solidaire à payer les sommes qui lui étaient demandées. Sous Louis XV et Louis XVI, les prélèvements s'espacent et prennent de nouvelles formes : en 1743, les trésoriers de Tours rachètent l'annuel pour 135000 l., ce qui n'empêche pas la monarchie, en 1771, de les soumettre au paiement du centième denier pour obtenir la suppression du prêt et du... droit annuel. Après une longue résistance, ils consentent finalement à payer 54000 l., faisant partie du million de livres exigés de l'ensemble des bureaux de finances du royaume. Comme cet exemple l'illustre, chaque exigence royale faisait l'objet d'âpres négociations qui étaient menées à l'échelon provincial par l'intendant au XVII ${ }^{\mathrm{e}}$ siècle puis qui furent prises en charge directement à Paris par les représentants du bureau. Pour réunir les capitaux qui leur étaient demandés, les trésoriers faisaient appel à différents bailleurs de fonds qui se recrutaient au sein de la compagnie et des élites tourangelles (mais très peu dans les communautés religieuses, à la différence de ce que l'on observe à Nantes), et, fait original, dans le monde des domestiques dans la deuxième moitié du XVIII ${ }^{\mathrm{e}}$ siècle. Du fait des prélèvements opérés, la compagnie a été victime d'un endettement chronique, de l'ordre de 300000 l., sans que cela ait affecté apparemment le patrimoine des officiers, comme le révèlent l'analyse des inventaires après décès conservés et celle des partages de succession, même s'il eût été nécessaire d'approfondir la réflexion sur ce point qui fait l'objet de débats entre les historiens spécialistes du monde des offices.

L'ouvrage se clôt par une analyse originale de l'action syndicale du bureau des finances de Tours à l'époque moderne (chapitre VIII). Connu pour la période de la Fronde, ce " lobbying " fait l'objet d'une analyse méthodique pour le XVIII ${ }^{\mathrm{e}}$ siècle. Grâce à l'étude des correspondances entre 
les officiers des différentes généralités et à la présentation de la stratégie suivie par leurs représentants, occasionnels ou permanents, à Paris, on se rend compte que les trésoriers ont âprement défendu leurs droits. Leur résistance victorieuse face aux entreprises menées contre leur juridiction par la Chambre des comptes ou le Parlement de Paris, l'obtention d'attributions en matière de voirie, sont dus en grande partie à cette intervention régulière auprès des organes centraux de gouvernement. Toutefois, la diversité des situations provinciales a rarement permis aux trésoriers de parler d'une seule voix et de d'imposer leurs vues au pouvoir central.

Au total, François Caillou nous présente un travail stimulant et bien écrit, qui, bien qu'il porte sur un sujet aride, n'est jamais ennuyeux. Il apporte une contribution appréciable aux enquêtes menées sur les officiers et sur les institutions d'Ancien Régime. On en retient que, sans être des officiers moyens du fait de leur privilège d'anoblissement graduel - que les conseillers au présidial n'ont jamais réussi à obtenir -, les trésoriers de France ne sont jamais parvenus, malgré leurs prétentions, notamment sur le plan fiscal (annuel, centième denier...), à être considérés sur le même pied que le personnel des cours souveraines. Rattachés à la Chambre des comptes de Paris où ils étaient reçus mais où ils ne siégeaient pas, ils ne formaient pas un véritable corps autonome, ce qui explique probablement leur incapacité à s'imposer dans leur ville d'exercice face aux autres institutions. Ils formeraient une catégorie à part que faute de mieux, François Caillou appelle « officiers supérieurs ». Leur déclin paraît toutefois incontestable malgré le plaidoyer qu'il dresse : la guerre de Trente ans leur a été fatale et dans ce sens, les trésoriers de France du XVIII ${ }^{\mathrm{e}}$ siècle n'ont pas grand-chose à voir avec leurs prédécesseurs. S'ils ont gardé une certaine vitalité, c'est surtout en se mettant au service des intendants, et en défendant des intérêts corporatistes. L'affaiblissement du pouvoir royal les a conduits à s'engager dans des conflits avec d'autres juridictions (parlement, Chambre des comptes...) mais sans leur permettre de retrouver de réelles prérogatives. S'ils ont pu prendre la responsabilité de la voirie secondaire, c'est parce que la monarchie ne s'y intéressait pas et qu'elle ne pouvait, si elle voulait maintenir le cours des offices, les priver de toute attribution. Ils n'avaient de toute façon pas la connaissance du terrain ni les compétences techniques pour assumer cette charge et, évoluant dans un cadre de plus en plus dépassé et contesté (offices, seigneuries...), il n'est guère surprenant qu'ils aient disparu au début de la Révolution française.

Sur le plan de la forme, on regrettera l'absence de véritable table des matières (celle qui est présentée s'apparente davantage à un sommaire) et une insuffisante subdivision du propos, particulièrement dans le chapitre $\mathrm{V}$ où l'on brasse beaucoup de thèmes qui auraient mérité d'être davantage mis en valeur. La réalisation d'un plus grand nombre de cartes (sur les origines géographiques des trésoriers, sur les réseaux de correspondance qu'ils entretenaient) et de graphiques (ex. sur l'évolution de l'endettement du bureau des finances) aurait permis d'alléger le texte. Quelques approximations de détail sont commises touchant notamment la cour dont il est 
dit qu'elle s'installe en Île-de-France à la fin des guerres de Religion - alors que le tournant a été pris dès le règne de François ${ }^{\text {er }}$, même si les troubles religieux ont provoqué un retour vers la vallée de la Loire - et la Fronde au cours de laquelle les officiers ne se révoltent pas seulement pour protester contre le non versement de leurs gages mais aussi et surtout contre la menace, autrement plus dangereuse, de suppression de l'annuel. Les jugements en matière d'ascension sociale ne tiennent pas suffisamment compte de l'idéal de vie des hommes de l'Ancien Régime : tous les officiers n'avaient sans doute pas l'ambition de progresser d'une génération à l'autre (p. 106); les trésoriers qui abandonnent toute activité après leur retrait du bureau sont considérés trop facilement comme des déclassés et les dérives comportementales de certains fils sont dépeintes, de façon un peu réductrice, comme des signes d'acculturation à la noblesse. On note ici et là des tendances à la digression avec des développements un peu longs sur les métiers et négoce de la soie, qui sont surtout pertinents pour les aïeux, sur la méthodologie de l'inventaire après décès ou du partage de succession. Il aurait fallu étudier de façon plus dynamique le marché de l'office (les traités mis à jour n'ont pas été systématiquement exploités) et mettre les différents aspects décrits en relation (en soulignant notamment le lien entre les exigences du pouvoir et l'évolution du prix des offices). Des familles et des lignages représentatifs comme les Sain, les Le Feuvre... auraient mérité une présentation plus détaillée. On en reste trop, conformément à la méthode prosopographique, à l'analyse du groupe alors que les archives notariales auraient permis de préciser les comportements des individus. De façon générale, on peut regretter que le second volume sur le personnel juxtapose des développements sans mettre suffisamment en avant la problématique défendue.

Malgré ces réserves, cette thèse ouvre des perspectives de recherche - sur le marché de l'office et des capitaux dans la France de l'Ouest; sur les bureaux de finances (il faudrait désormais s'intéresser à ceux des pays d'états qui sont les plus mal connus), sur la notion de service du roi... - et à ce titre, sa lecture est à conseiller. 